\title{
Oil Well Characterization and Artificial Gas Lift Optimization Using Neural Networks Combined with Genetic Algorithm
}

\author{
Chukwuka G. Monyei, ${ }^{1}$ Aderemi O. Adewumi, ${ }^{2}$ and Michael O. Obolo ${ }^{3}$ \\ ${ }^{1}$ Department of Electrical and Electronic Engineering, University of Ibadan, Nigeria \\ ${ }^{2}$ School of Mathematics, Statistics and Computer Science, University of KwaZulu-Natal, South Africa \\ ${ }^{3}$ Department of Petroleum Engineering, University of Ibadan, Nigeria
}

Correspondence should be addressed to Aderemi O. Adewumi; laremtj@gmail.com

Received 24 January 2014; Revised 23 March 2014; Accepted 8 April 2014; Published 22 May 2014

Academic Editor: Weihua Liu

Copyright (C 2014 Chukwuka G. Monyei et al. This is an open access article distributed under the Creative Commons Attribution License, which permits unrestricted use, distribution, and reproduction in any medium, provided the original work is properly cited.

\begin{abstract}
This paper examines the characterization of six oil wells and the allocation of gas considering limited and unlimited case scenario. Artificial gas lift involves injecting high-pressured gas from the surface into the producing fluid column through one or more subsurface valves set at predetermined depths. This improves recovery by reducing the bottom-hole pressure at which wells become uneconomical and are thus abandoned. This paper presents a successive application of modified artificial neural network (MANN) combined with a mild intrusive genetic algorithm (MIGA) to the oil well characteristics with promising results. This method helps to prevent the overallocation of gas to wells for recovery purposes while also maximizing oil production by ensuring that computed allocation configuration ensures maximum economic accrual. Results obtained show marked improvements in the allocation especially in terms of economic returns.
\end{abstract}

\section{Introduction}

Petroleum, a limited natural resource, is a nonrenewable form of energy on which humans largely depend. This leads to pressing market demands, accessibility issues, and competitive market environment that force oil companies to seek technologies and procedures that can give competitive advantage and meet environmental restrictions while streamlining production processes and cutting costs [1].

Artificial gas lift (AGL) is a recovery process that involves the use of gases, produced (from oil) or purchased, which are pumped into the well bore to maintain formation pressure, that is, the pressure at which the fluid flows to the surface. There are two types of AGL, namely, intermittent gas lift and continuous gas lift [1]. However, this paper is not concerned with the types of AGL but rather its distribution or allocation.

The gas lift process involves the injection of high pressure gas at the bottom of the production tubing of an oil well [13]. In other words, AGL involves injecting high-pressured gas from the surface into the producing fluid column through one or more subsurface valves set at predetermined depths $[2,3]$. This helps to improve recovery by reducing the bottom-hole pressure at which wells become uneconomic, resulting in being abandoned. The gas, mixed with the oil, diminishes the weight of the fluid column thereby reducing the downhole pressure. A low downhole pressure induces a flux of fluids from the reservoir to the well. The produced fluid is composed of oil, gas, and water. The water must be treated before being discharged which incurs costs while the gas can be either reused in the process or sent to customers and other facilities [3]. In large oil fields, several separators are used to divide the three phases. This gives rise to the problem of maximizing production by allocating lift-gas to the wells while defining the routing from wells to separators and observing separator capacities $[2,3]$.

In particular, the gas lift operation of oil fields is one of many production processes whose performances can be improved. As the internal pressure in high-depth or depleted reservoirs can force the flow of only a fraction of their oil to the surface, the use of artificial means becomes imperative 
to lift the oil, especially for deep reservoirs that are found off-shore. Two examples of artificial lifting are submerged pumps and continuous injection of gas [3]. Although the former can, in principle, recover most of the oil, its operating costs are excessively high for today's oil prices, not to mention the potential of an unfavourable energy trade and other technical hindrances. The gas lift technique, on the other hand, harnesses the reservoir's gas by injecting natural gas into the production tubing so as to reduce the weight of the oil column, thereby elevating the mix of oil, gas, and water to the surface.

A motivation for this work therefore arises from the need to reduce wastage in gas allocation especially in the unlimited scenario thus freeing up gas thus freeing up gas for other uses such as domestic, transportation and electricity generation purposes. With global outcry to the insidious effect of green-house gases on our environment and the need for prudent management of scarce resources, this paper aims at providing a cost-effective method for solving the problem of gas allocation for recovery purposes in the oil and gas industry.

The rest of this paper is organized as follows: Section 2 presents a brief overview of related works in literature while Section 3 further describes the problem as well as modelling approach. Section 4 gives an overview of the methodology adopted to solve the oil well characterization and AGL problem while results obtained are discussed in Section 5. The final section presents some useful conclusions as well as direction for further works.

\section{Literature Review}

Gas injection has been used to maintain reservoir pressure at some selected levels or to supplement natural reservoir energy to a lesser degree by reinjection of a portion of the produced gas. Complete or partial pressure maintenance operations can result in increased hydrocarbon recovery and improved reservoir production characteristics. A general position opines that daily oil production increases concomitantly with gas up to a certain level where further gas injection yields a decrease in oil production with increased gas cost [2]. Ray and Sarker [2] developed a multiobjective constrained algorithm to optimize gas lift allocation within the constraint of limited available gas. The proposed solution was applied to six and fifty-six well problems with single and multiobjective problem formulations. De Souza et al. [4] described a case study that involved modelling and optimizing gas allocation for deep water offshore petroleum production with interest in determining the rate of injected gas flow that guarantees maximum oil production, profit, and optimal design of gas lift system considering capital cost of compressors, turbine, and gas pipeline constraints. The problem was modelled as a nonlinear optimization problem and solved as a two-phase network flow model. Codas and Camponogara [1] addressed the problem of gas lift allocation with separator routing constraint using a mixed integer linear model solved using the CPLEX software. Mahmudi and Sadeghi [5] used a hybrid computational model consisting of genetic algorithm (GA) and Marquardt algorithm to optimize gas allocation under various constraints including effects of tubing diameter, rates of gas injection, and separator pressure on the economic return of the well over a long period.

From the foregone and other research trends, the problem of well characterization and gas allocation is mainly addressed independently. Moreover, specific interests of earlier works did not focus on configuration selection but rather on routing mechanisms such as separator routing constraint, effects of tubing diameter, rates of gas injection and separator pressure on the economic return of the well over long period, and capital cost of compressors, turbine, and gas pipeline amongst others. Most previous works therefore focused on the contribution of the artificial gas lift layout and material selection in ensuring optimum gas yield. This paper seeks to complement ongoing research by proposing a combined modified artificial neural network (MANN) and mild intrusive genetic algorithm (MIGA) intelligent technique for optimum well characterization and gas lift allocation to achieve maximum economic yield. We seek to characterize the oil wells and get maximum produced oil using limited available gas which indirectly results in increased economic returns. Further details and description of the gas lift problem can be found in [2-4, 6-8].

\section{Problem Definition}

3.1. Statement of the Problem. An oil field consisting of six wells is considered in this paper. AGL is employed in improving the recovery of the respective wells. Available gases at times are not sufficient to guarantee maximum oil production from the wells while classical techniques also do not guarantee optimal allocation of these available gases; hence an efficient algorithm that can optimally allocate them by selecting the best configuration that can ensure optimum economic accrual is sought. This paper proposes the MANNMIGA approach for this problem.

3.2. Modelling. This paper addresses a case scenario involving six wells and limited gas supply that is currently not able to guarantee maximum production. We employ modified versions of artificial neural networks (ANN) and GA which has been successfully used in literature (e.g., see $[9,10])$. These algorithms seek to optimize the allocation of available gas quantity among the six wells under consideration by allocating quantities that guarantee maximum economic accrual. First, MANN is used to characterise the oil production in terms of $\mathrm{B} / \mathrm{D}$ (B/D refers to unit of measuring oil production output in terms of barrels per day) and the capacity of each well with respect to the gas injected (in MMscf/D), MMscf/D refers to unit of measuring gases in terms of million standard cubic feet per day. Next, MIGA is used to select the best values for each oil well while observing the following.

(i) Total gas allocated for the six wells does not exceed the available gas quantity for optimization.

(ii) Economic accrual is more important than allocating entire gas and must therefore be maximized. 
(iii) Where there are two feasible values for gas allocation with the same oil production, the lowest value is chosen.

The work seeks to address the issue of efficient usage of scarce gas thus preventing wastage and enhancing oil production. In the event of other uses of gas emanating, optimization of the available gas for optimum oil production, maximum economic accrual, and other emanating needs is possible.

The main objective is to develop a strategy for the optimal allocation of limited (and unlimited) gas supply in an oil field involving six wells. Gas allocation is to be minimized while profit from both the sale of oil and remaining unallocated gas is to be maximized by maximizing oil production. We assume full dispatch of gas thus neglecting interaction between gas, conveying media, and other intermeddling media.

If $i$ represents the index of the wells and $n$ is the number of wells, the objective function is formulated as follows

$$
\begin{aligned}
& \text { Minimize gas allocation }=\sum_{i=1}^{n}\left[G_{u}(\cdot)\right] \\
& \text { Maximize oil production }=\sum_{i=1}^{n}\left[O_{u}(\cdot)\right] \\
& =\sum_{i=1}^{n}\left[a+b G_{u}(\cdot)\right. \\
& \left.\quad+c G_{u}(\cdot)^{2}+d G_{u}(\cdot)^{3}\right],
\end{aligned}
$$

Maximize profit from sales $=P_{g} \sum_{i=1}^{n}\left[O_{u}(\cdot)\right]+P_{o} O_{m}$,

where $G_{u}(\cdot)$ is gas unit allocated for well $I ; O_{u}(\cdot)$ is respective oil produced for respective gas allocated to well $i ; a, b, c$, and $d$ are constants for derived polynomial function and $P_{g}$ is unit price of gas $(\$ / B) ; P_{o}$ is unit price of oil (\$/MMscf); $O_{m}$ is unallocated gas $=T_{a}-\sum_{i=1}^{n}\left[G_{u}(\cdot)\right] ; T_{a}$ is (un)limited gas available for allocation.

Subject to

(1)

$$
\sum_{i=1}^{n}\left[G_{u}(\cdot)\right] \leq T_{a} \gamma i
$$

(2) $i$, if $G_{i}$ is $a$ or $b$ and $G_{i}(a)=G_{i}(b)=O_{i}$, where $O_{i}$ is oil produced for $G_{i}(\cdot)$, if $a<b$ gas allocated $=G_{i}(a)$.

Also, the equation governing gas allocation (MMscf/D) and oil produced $(\mathrm{B} / \mathrm{D})$ is given as

$$
\mathrm{Q}_{\mathrm{Li}}=a \mathrm{Q}_{\mathrm{Q} i}^{2}+b \mathrm{Q}_{\mathrm{Q} i}+c
$$

where $i$ is well number, $Q_{L i}$ is oil produced per well (in $\mathrm{B} / \mathrm{D}$ ), $\mathrm{Q}_{\mathrm{Q} i}$ is gas allocated (in MMscf/D), and $a, b$, and $c$ are constants

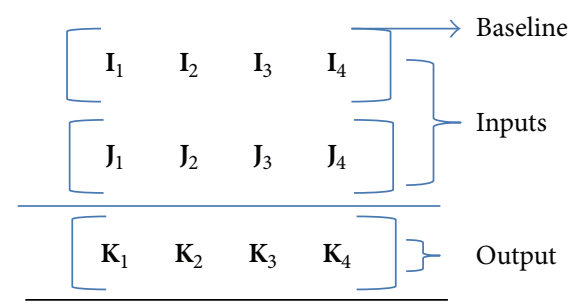

FIGURE 1: Received inputs and output during training.

TABLE 1

\begin{tabular}{lccc}
\hline Well & Value $a$ & Value $b$ & Value $c$ \\
\hline 1 & 1138.71 & 799.94 & -284.00 \\
2 & 841.34 & 893.50 & -277.69 \\
3 & 131.28 & 61.54 & 0 \\
4 & 135.92 & 39.08 & 0 \\
5 & 125.38 & 49.05 & 0 \\
6 & 156.35 & 89.46 & 0 \\
\hline
\end{tabular}

The value of the constants $a, b$, and $c$ for the six wells is given in Table 1.

The values used in training the MANN (Section 4) were obtained from [8].

\section{Methodology}

As stated earlier, this work adopts combined algorithms of MANN-MIGA which are essential modified forms of ANN and GA. The choice lies in the facts that the underlying techniques have been successfully used in other similar problems as they offer needed efficiency, speed, and flexibility $[6,7]$. Moreover, the combination of MANN and MIGA has been efficiently used to solve other problems in literature with promising results $[9,10]$. We present below a detailed description of the methods as applied to the current problem.

4.1. Modified Artificial Neural Network. In characterizing the gas injection/oil production of the wells, MANN was used in generating a model. In designing the algorithm for the neural network, a simple regression formula was used. The algorithm receives the inputs, sorts them out, adjusts its parameters, and computes the expected result. A step by step overview of the algorithm is presented as follows.

(1) The inputs are received in the proper (same) dimension (Figure 1).

(2) Interpolation is then carried out on each input matrix and each output matrix using their respective minimum and maximum values in generating an equivalent value of the contribution of each input to the expected answer or result.

(3) Received interpolated input(s) and output(s) are sorted (ranked) concurrently in order to obtain a minimum and maximum value with the first matrix serving as a baseline (Figure 2). 


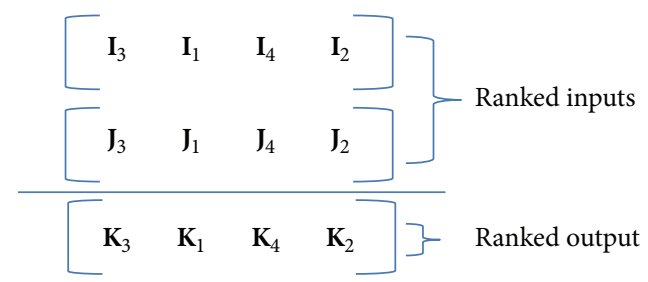

FIGURE 2: Ranked received inputs and output.

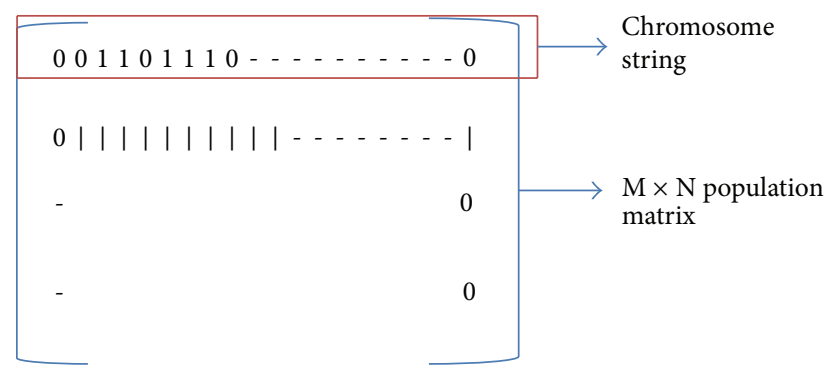

FIgURE 3: Sample space for MIGA.

(4) Since MATLAB makes use of matrices, received inputs are thus stored in matrices. The output matrix is checked for crossovers (transition from a high-lowhigh or low-high-low number) using the first input matrix as baseline.

(5) The transitions obtained from the output matrix are used in generating the order of the polynomial function or curve in which our data is to be fitted into (5)-(10). As can be surmised from (5)-(10), there are two inputs and one output (see Figure 1). The modelling of the first input is calculated using (5)(7) which are used in generating the respective values of $a, b$, and $c$. Our first output is thus given as $\mathbf{K}(\mathbf{I})=a \mathbf{I}^{\mathbf{2}}+b \mathbf{I}+c$. The second input is modeled similarly using (8)-(10) which are used in generating the respective values of $a 1, b 1$, and $c 1$. The second output is thus given as $\mathbf{K}(\mathbf{J})=a 1 \mathbf{J}^{2}+b 1 \mathbf{J}+c 1$. Our final output is thus the average of these two values given as $\mathbf{K}=(\mathbf{K}(\mathbf{I})+\mathbf{K}(\mathbf{J})) / 2$.

(6) Generated value(s) is/are then recalculated in order to obtain actual values which are then fed forward to the output.

(7) Before the generated values are displayed, they are adjusted for errors using appropriate weights. For the purpose of this analysis, the weights have been assumed to be unity and there is no back propagation network provided to assist in adjusting the displayed values. The inputs therefore must be reliable and fairly accurate.

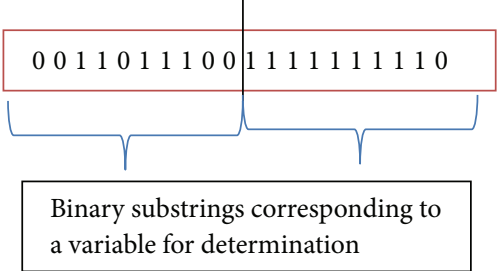

Figure 4

If

$$
\begin{aligned}
& \mathbf{K}_{1}>\mathbf{K}_{3} \\
& \mathbf{K}_{1}>\mathbf{K}_{4} \\
& \mathbf{K}_{2}<\mathbf{K}_{4},
\end{aligned}
$$

where $\mathbf{I}_{i}, \mathbf{J}_{i}$, and $\mathbf{K}_{i}(i=1,2,3,4)$ are valid inputs and output, respectively, then the number of crossovers could be determined from the ranked output. From (1) therefore, the ranked output of Figure 2 has 1 crossover.

One crossover therefore signifies that our data could be modeled using a polynomial of order 2 (i.e., a quadratic equation). In modelling therefore the following equations are used:

$$
\begin{gathered}
\sum \mathbf{K}_{i}=a * n+b \sum_{i=1}^{n} \mathbf{I}_{i}+c \sum_{i=1}^{n} \mathbf{I}_{i}{ }^{2}, \\
\sum \mathbf{K}_{i} \mathbf{I}_{i}=a \sum_{i=1}^{n} \mathbf{I}_{i}+b \sum_{i=1}^{n} \mathbf{I}_{i}{ }^{2}+c \sum_{i=1}^{n} \mathbf{I}_{i}{ }^{3}, \\
\sum \mathbf{K}_{i} \mathbf{I}_{i}{ }^{2}=a \sum_{i=1}^{n} \mathbf{I}_{i}{ }^{2}+b \sum_{i=1}^{n} \mathbf{I}_{i}{ }^{3}+c \sum_{i=1}^{n} \mathbf{I}_{i}{ }^{4}, \\
\sum \mathbf{K}_{i}=a 1 * n+b 1 \sum_{i=1}^{n} \mathbf{J}_{i}+c 1 \sum_{i=1}^{n} \mathbf{J}_{i}{ }^{2}, \\
\sum \mathbf{K}_{i} \mathbf{J}_{i}=a 1 \sum_{i=1}^{n} \mathbf{J}_{i}+b 1 \sum_{i=1}^{n} \mathbf{J}_{i}{ }^{2}+c 1 \sum_{i=1}^{n} \mathbf{J}_{i}{ }^{3}, \\
\sum \mathbf{K}_{i} \mathbf{J}_{i}{ }^{2}=a 1 \sum_{i=1}^{n} \mathbf{J}_{i}{ }^{2}+b 1 \sum_{i=1}^{n} \mathbf{J}_{i}{ }^{3}+c 1 \sum_{i=1}^{n} \mathbf{J}_{i}{ }^{4} .
\end{gathered}
$$

4.2. Mild Intrusive Genetic Algorithm. MIGA is used in combination with the MANN in arriving at optimum solutions for gas allocation under limited and unlimited conditions. GA is an evolutionary algorithm that mimics the principle of natural selection, reproduction, and survival of the fittest in solving complex optimization problems [11]. It has been widely and successfully used in literature howbeit in modified or improved form from the standard GA [11, 12] when it comes to some complex problems [13-15]. GA has also been applied to difficult problem involving the control of gas pipeline transmission $[6,7]$.

MIGA is designed as a modified population-based technique. Figure 3 provides a sample space that illustrates the 


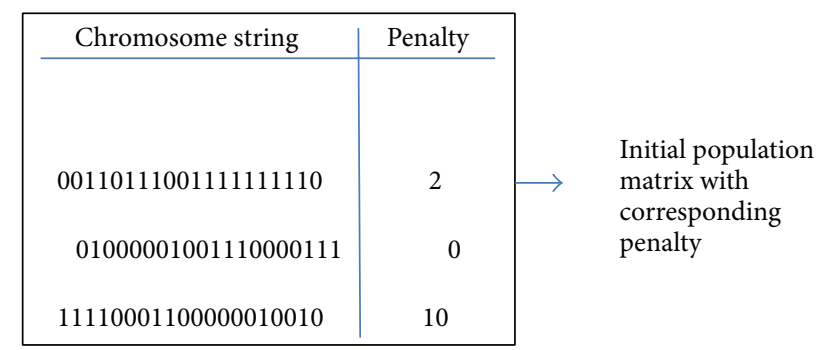

(a)

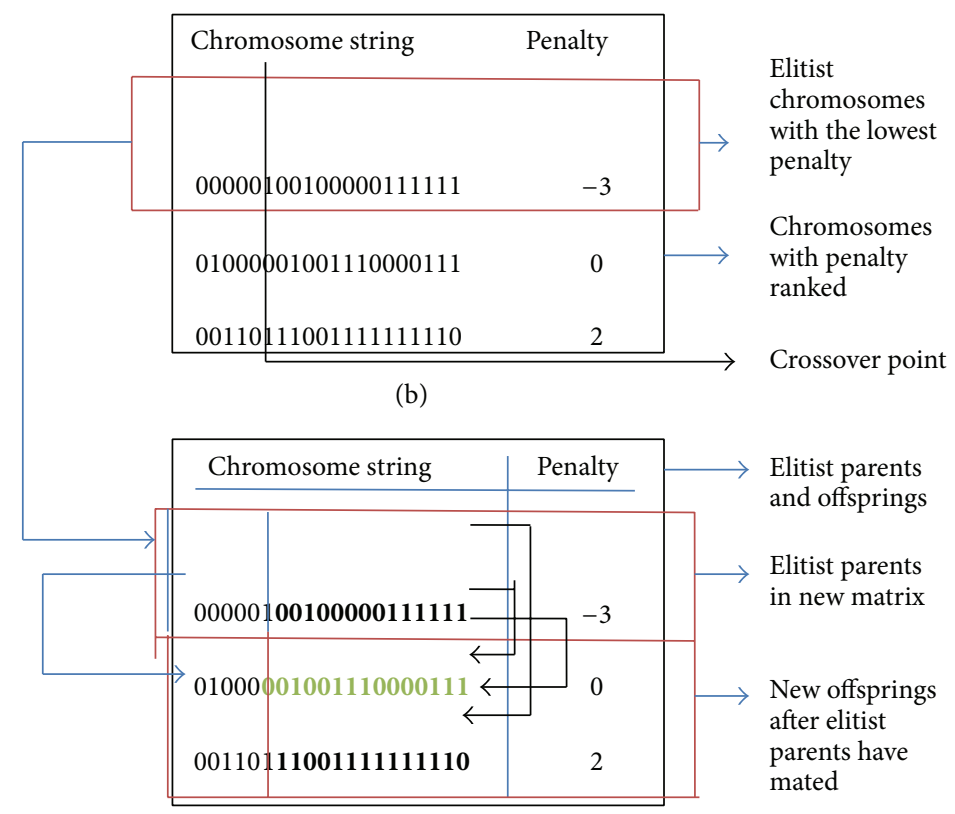

(c)

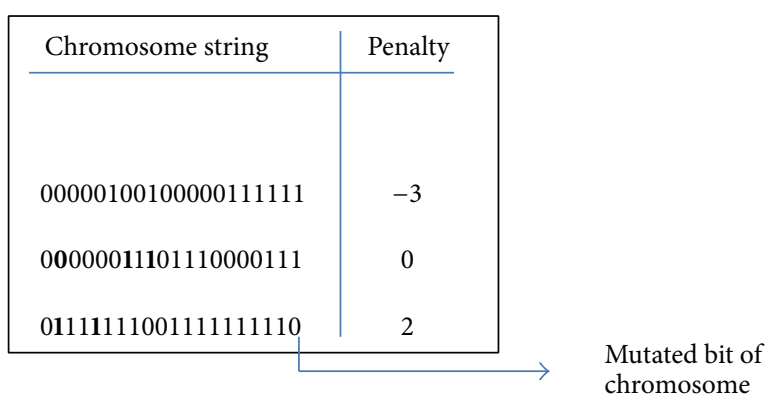

(d)

FIGURE 5: Illustration of MIGA steps.

environment for the activities of MIGA. GA allows for various ways of defining the population structure (chromosome) $[14,16]$.

In this study, MIGA uses the binary representation. It then runs through the usual steps of selection, crossover, elitism, and mutation as illustrated in Figure 5. Each chromosome string (Figure 4) corresponds to a solution whose fitness is tested for optimality (fitness value of zero). Figure 5(b) shows an instance of a poor solution with fitness value farther from zero.

\section{Simulation Experiment, Results, and Discussions}

5.1. Simulations Environments. Simulation experiment for this work was done with MATLAB R2009a. Results were interpreted in form of graphs (as shown in Figures 6-13) and tables (as depicted in Tables 2 and 3 ) generated within this environment. The figures are grouped sometimes into three groups based on the well allocation number as presented and briefly discussed below. Experiment was done on a system 


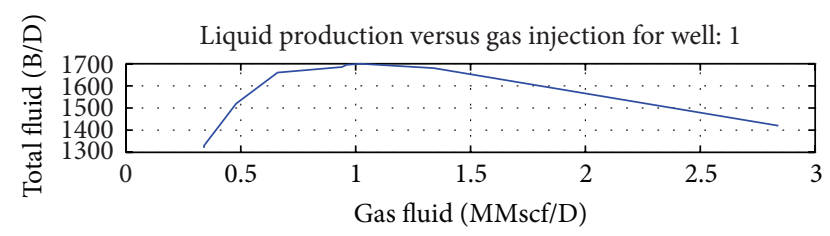

(a)

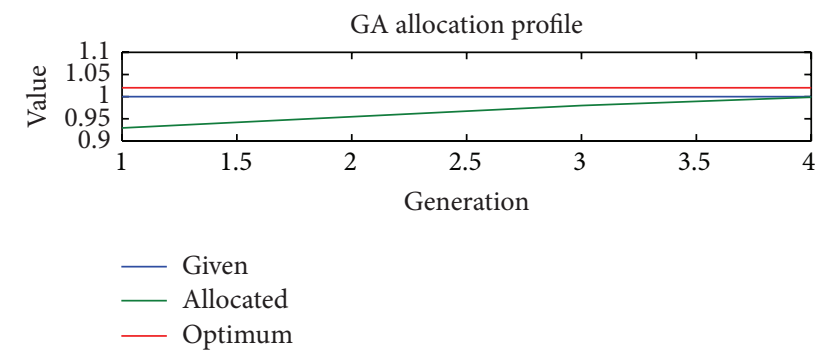

(b)

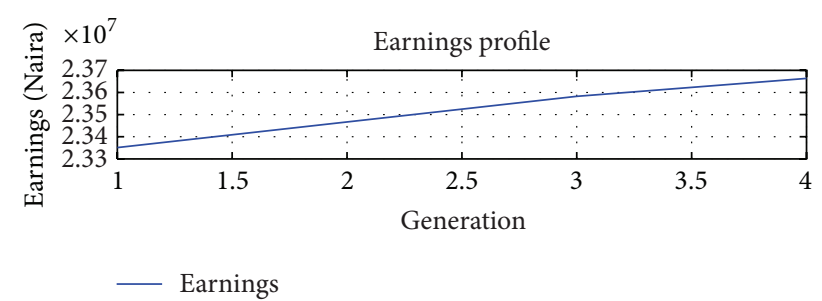

(c)

FIGURE 6: Well 1's allocation profile, GA allocation profile, and the earnings (Naira) profile for a limited scenario.

with 4 GB DDR3 Memory, 500 GB HDD, and an Intel Core CM $^{T M}$ i3-380 M Processor.

5.2. Results and Discussion. Based on the application of the proposed methodology in Section 4, results obtained for gas allocation to the six wells under the limited and unlimited scenario are presented mainly in graphical forms as given in Figures 6-13. The behaviour of the proposed MANN-MIGA in effectively characterizing and allocating gas is discussed subsequently. Also discussed is the economic accrual obtained by methodology for different allocation configuration within defined generation run. Tables 2 and 3 show the best allocation values for the limited and unlimited scenario and also the average allocation.

Figure 6(a) displays the curve establishing the relationship between the allocated gas (in MMscf/D) and the oil produced (in $\mathrm{B} / \mathrm{D}$ ). It will be noted in this figure that the allocation of gases does not exceed the maximum point on the curve as already defined in given constraints otherwise it would have led to wastage since increasing allocation beyond the maximum point yields reduced oil production. The curve displayed in Figure 6(a) also echoes with the curves displayed in Figures 7(a), 8(a), 9(a), 10(a), 11(a), 12(a), and 13(a). The curves establishing the gas allocated versus oil produced for wells 1, 2, 3, and 6 are displayed in Figures 6(a) and 7(a), Figures 8(a) and 9(a), Figures 10(a) and 11(a), and Figures 11(a) and 12(a), respectively. It is observed that results

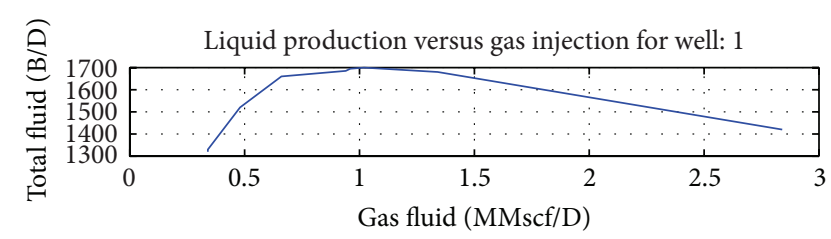

(a)

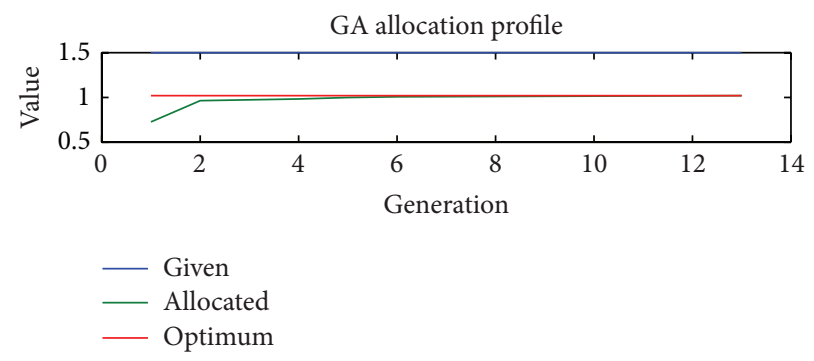

(b)

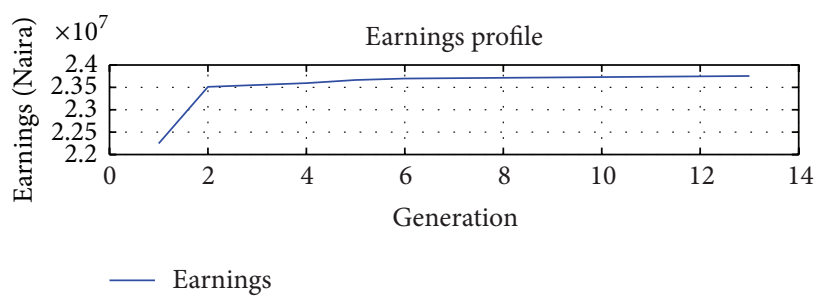

(c)

Figure 7: Well 1's allocation profile, GA allocation profile, and earnings (Naira) profile in an unlimited scenario.

illustrated in these graphs satisfy the expected constraints; that is, the allocation of gases does not exceed the value corresponding to the maximum oil that could be produced from that well.

In an attempt to allocate gas under the limited scenario in order to achieve maximum economic yield, Figures 6(b), $8(\mathrm{~b}), 10(\mathrm{~b})$, and 12(b) show how best gas was distributed among wells $1,2,3$, and 6 , respectively. The available gas in each of the considered scenarios was less than the sum of the gas value(s) corresponding to peak oil production for the number of wells under consideration. The MANNMIGA results shown in Figures 6(b), 8(b), 10(b), and 12(b) in allocating gas present the obtained best configuration that ensures maximum economic yield. The economic yield being referred to includes the combined accrual from sales of the respective oil produced and the unallocated gas using current market values.

Similarly, the combined MANN-MIGA was also used in allocating gas during an unlimited scenario (where the available gas for allocation exceeds the combined sum of the gas value(s) corresponding to peak oil production for the number of wells under consideration). Figures 7(b), 9(b), 11(b), and 13(b) show the allocation profiles for 1, 2, 3, and 6 wells during an unlimited scenario. Figures 7(b), 9(b), 11(b), and 13(b) display the earnings based on the MANN-MIGA values and current market values for gas and oil. 
TABLE 2: Limited gas optimization values for different well combinations.

\begin{tabular}{lcccc}
\hline Well number & 1 & $1-2$ & $1-3$ & \\
\hline Optimum value (MMscf/D) & 1.02 & 2.16 & 3.29 & 7.71 \\
\hline Given value (MMscf/D) & 1 & 2 & 3 & Well 1: 0.75867 \\
\hline & & & Well 2: 0.78643 & Well 3: 0.75565 \\
Allocated (MMscf/D) & Well 1: 0.999 & Well 1: 0.966 & Well 4: 0.50765 & Well 5: 1.2298 \\
& & Well 2: 1.0324 & Well 2: 1.0285 & Well 6: 0.96 \\
\hline Unallocated (MMscf/D) & & & & 0.0018 \\
\hline allocation & 0.001 & 0.001 & 0.00037 & 99.96 \\
\hline
\end{tabular}

TABLE 3: Unlimited gas optimization values for different well combinations.

\begin{tabular}{lcccc}
\hline Well number & 1 & $1-2$ & $1-3$ & $1-6$ \\
\hline Optimum value (MMscf/D) & 1.02 & 2.16 & 3.29 & 7.71 \\
\hline Given value (MMscf/D) & 1.5 & 3 & 5 & Well 1: 0.81467 \\
\hline & & & Well 2: 1.0593 & Well 3: 1.0592 \\
Allocated (MMscf/D) & Well 1: 1.02 & Well 1: 1.0173 & Well 4: 1.99 & Well 5: 1.4112 \\
& & Well 2: 1.14 & Well 2: 1.14 & Well 6: 0.99554 \\
\hline Unallocated (MMscf/D) & & & & 1.38 \\
\hline \% allocation & nil & & & 0.024 \\
\hline
\end{tabular}

In summary, four scenarios were considered for two conditions in each scenario, that is, limited and unlimited gas availability. The first scenario involved well 1 only, second scenario involved wells 1 and 2 only, and third scenario involved 3 wells, 1, 2, and 3 only, while fourth scenario involved the six wells. In each scenario, MANN-MIGA was tested for both limited and unlimited gas (in MMscf/D) availability. Figures 6(b), 8(b), 10(b), and 12(b) show the allocation profile of MANN-MIGA in the limited scenario. As can be observed, gas quantity available for allocation, that is, given quantity (blue line), is less than the optimum value (red line) needed for maximum oil production. The allocation profile (green line) traces the given profile (blue line) in maximizing oil production. It should be noted that, in maximizing oil production and earnings (as shown in Figures $6,8,10$, and 12 ), the criticality of the allocation configuration cannot be overemphasized. In essence, the configuration with the highest earnings is chosen.

Similarly, MANN-MIGA was also tested for the unlimited condition. As can be observed, Figures 7(b), 9(b), 11(b), and 13(b) show the allocation profile under this condition. A further observation of the aforelisted figures shows that the given quantity (blue line) is greater than the optimum value (red line) needed for maximum oil production. Gas allocation (green line) is thus expected to follow the optimum value (red line) in generating maximum oil production values. The earning profiles for the different considered scenario under the unlimited condition are shown in Figures 7(c), 9(c), 11(c), and 13(c). In generating allocation configuration values and earnings, an oil price of $\mathrm{N} 14,000 / \mathrm{B}$ (Naira (N) is the currency for Nigeria where the work was carried out and based. The displayed value is in terms of Naira per barrel) and gas price of $\$ 400 / M M s c f$ were used.

The subsequent tables show the new values obtained in both the limited and unlimited scenarios. A critical observation of Figures 6(b), 8(b), 10(b), and 12(b) for the limited scenario and Figures 7(b), 9(b), 11(b), and 13(b) for the unlimited scenario reveals some deviations. As presented in Table 2, the MANN-MIGA under the limited scenario has a better allocation (about 99.94 on average) compared to the MANN-MIGA allocation values presented in Table 3 during the unlimited scenario (about 95.31 on average). As can be observed from Tables 2 and 3, three allocation terms are presented as further shown in the earlier considered figures. They are the optimum, given, and allocated values. The optimum value shows the maximum value that can be allocated to the well(s) under consideration guaranteeing the maximum oil that can be produced. The given value denotes the scenario under consideration, limited or unlimited, while the allocated value describes the ability of the MANNMIGA in optimally distributing the given value for optimal economic/monetary yield. Our algorithm is therefore able to characterize the wells using a least square approximation method embedded into MANN and also allocate gas to the well combinations under consideration during limited (over $99 \%$ on average) and unlimited scenario (over $95 \%$ on 


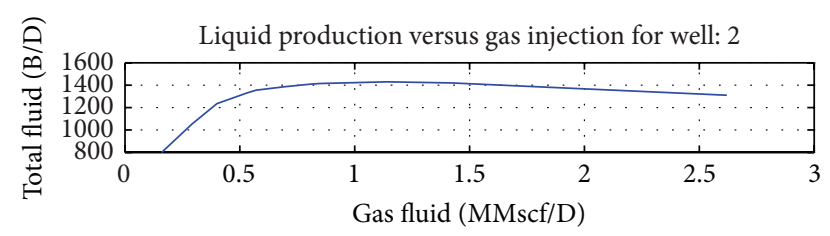

(a)

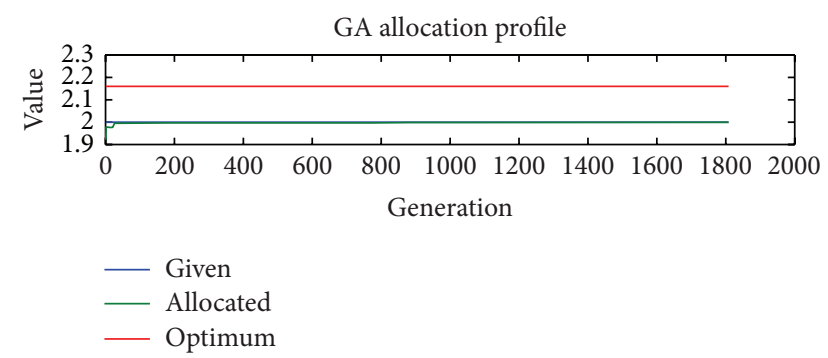

(b)

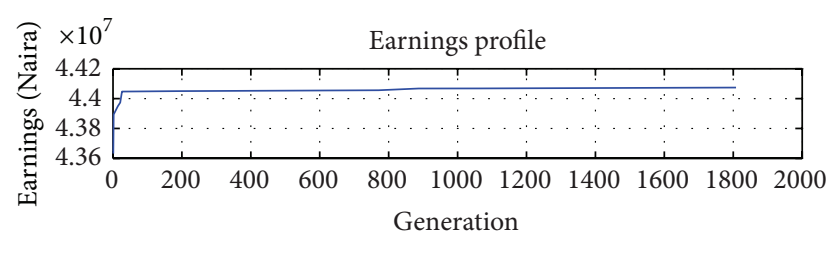

— Earnings

(c)

FIGURE 8: Well 2's allocation profile, wells 1-2 GA allocation profile, and wells 1-2 earnings (Naira) for a limited scenario.

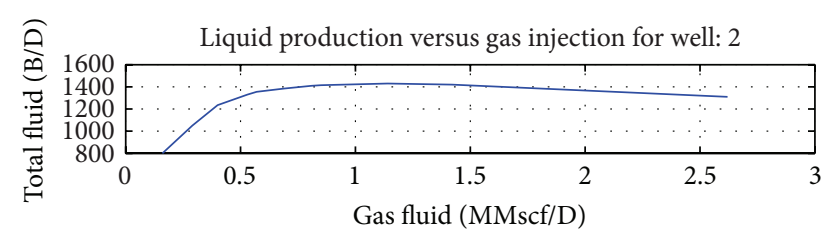

(a)

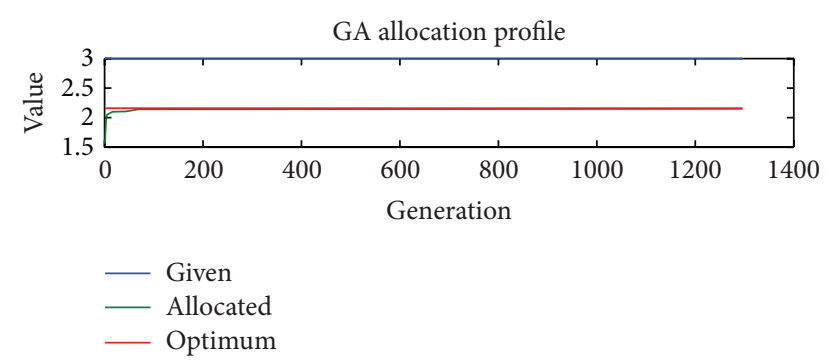

(b)

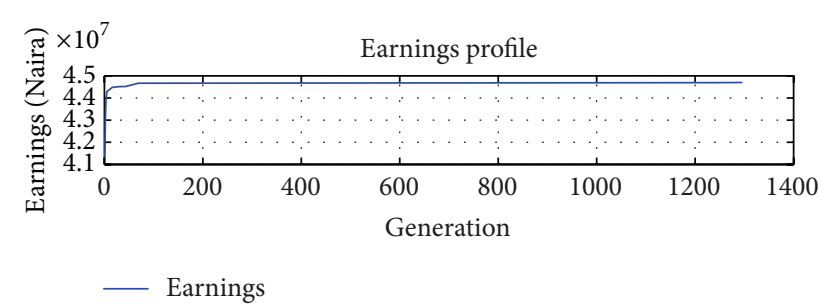

(c)

FIGURE 9: Well 2's allocation profile, wells 1-2 GA allocation profile, and wells 1-2 earnings (Naira) in an unlimited scenario.

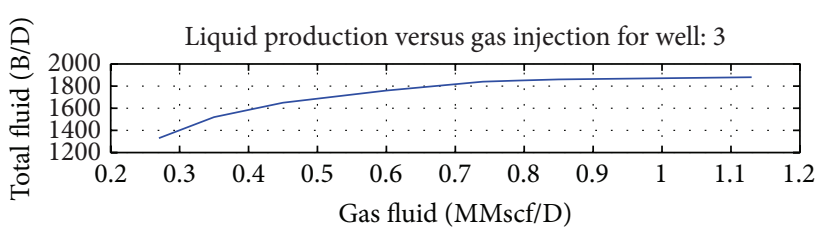

(a)

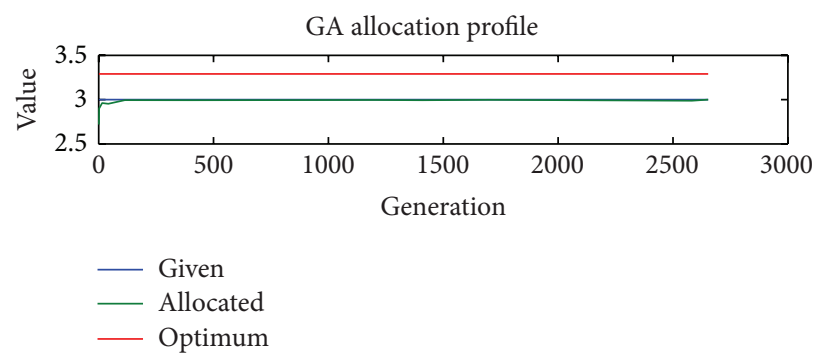

(b)

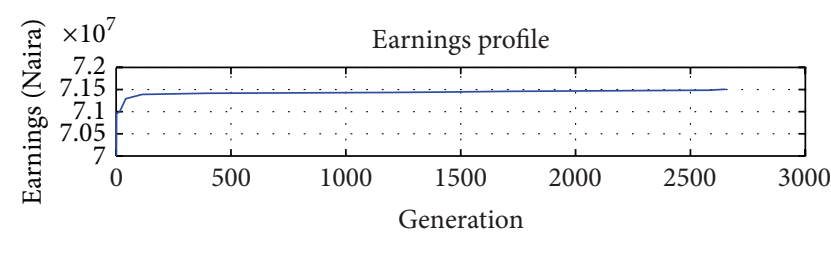

(c)

FIGURE 10: Well 3's allocation profile, wells 1-3 GA allocation profile, and earnings (Naira) in a limited scenario.

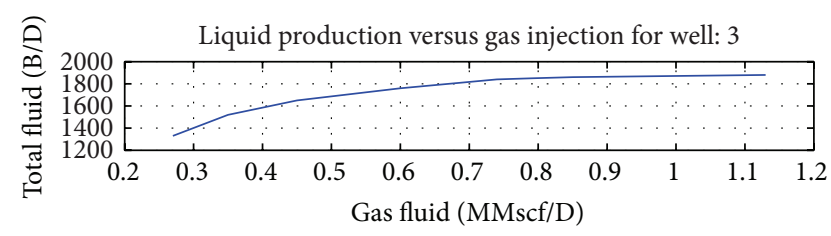

(a)

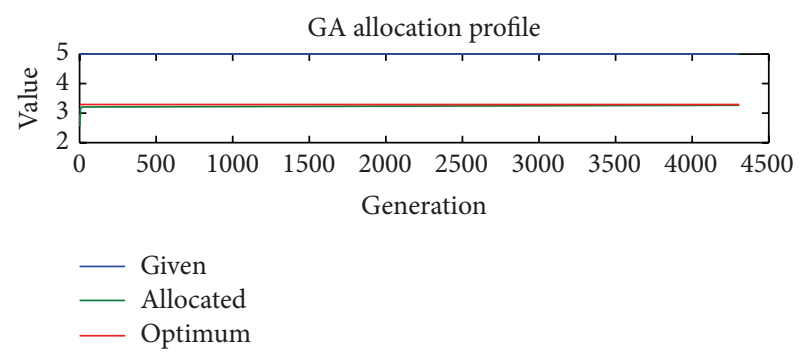

(b)

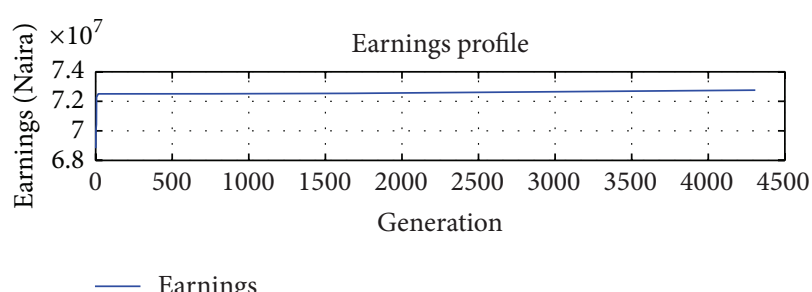

(c)

FIGURE 11: Well 3's allocation profile, wells 1-3 GA allocation profile, and earnings (Naira) in an unlimited scenario. 


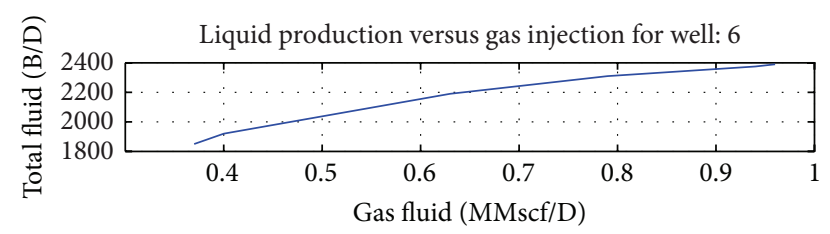

(a)

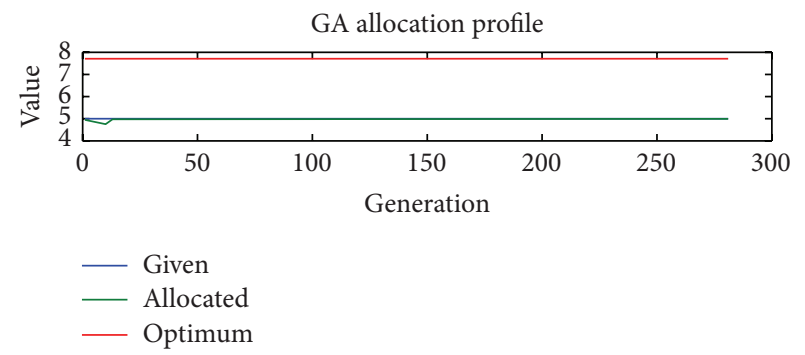

(b)

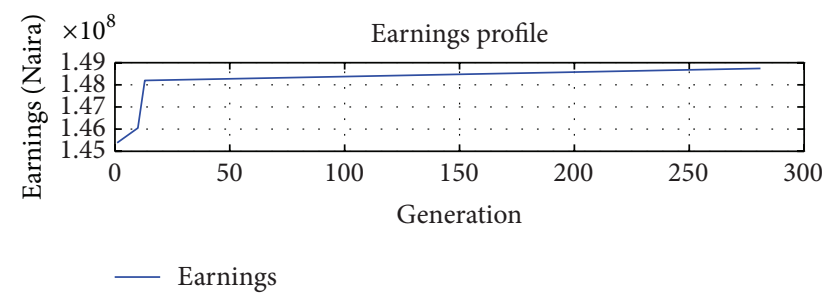

(c)

FIGURE 12: Well 6's allocation profile, wells 1-6 GA allocation profile, and earnings (Naira) for a limited scenario.

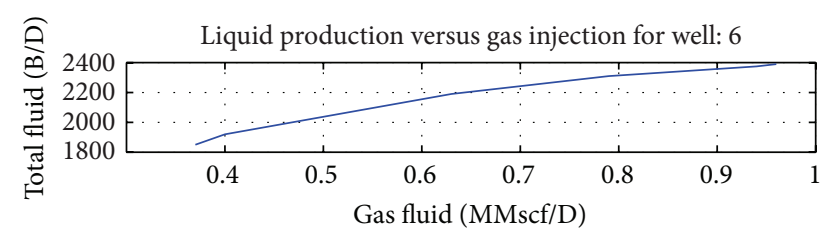

(a)

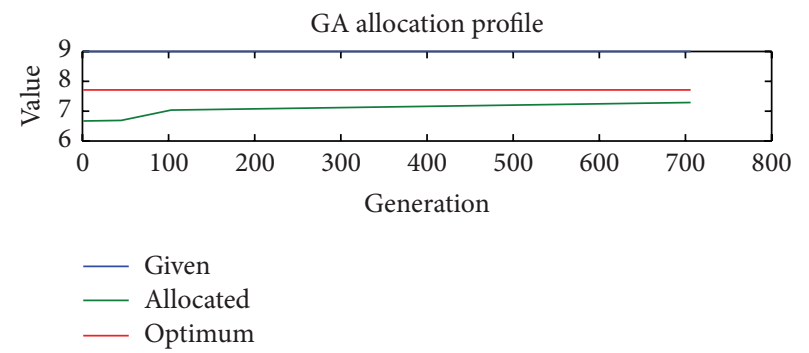

(b)

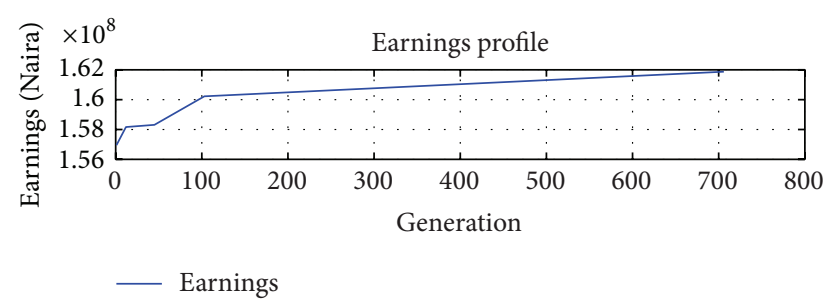

(c)

FIGURE 13: Well 6's allocation profile, wells 1-6 GA allocation profile, and earnings (Naira) for an unlimited scenario. average) using the MIGA as a stochastic optimization tool. The algorithm provided consistent results on multiple runs and at a fast run time.

\section{Conclusion and Future Work}

This paper presents a characterization of oil wells and gas allocation in both the limited and unlimited scenarios such as presenting a cost-effective means for gas allocation for the oil and gas industry. While earlier works considered the characterization and allocation separately, this work has been able to both characterize and use generated values from the characterization in allocating gas. A mathematical model is obtained for characterizing the gas allocated and oil produced for the wells under consideration. Wells 1 and 2 were characterized using a quadratic equation while the rest were subsequently characterized using linear curve fitting techniques. A combined MANN-MIGA was adapted and applied extensively in evolving a relationship between the gas allocated and oil produced for the six wells considered. These values have been further used in computing the optimum gas allocation per well. The values generated show a remarkable allocation by our approach. The mild intrusive property of our GA arises from its ability to allocate the least possible gas value for maximum oil production. The approach neglects gas values that exceeded the maximum for optimal oil production. The MIGA was then used in generating optimized values which met given conditions and yielded improved economic returns. The MANN-MIGA thus proves useful to the oil and gas industry as it not only provides characterized equations, but also allocates gas based on preset conditions.

Future work might consider further fine-tuning of the algorithm for improved performance especially in optimizing gas allocation under the unlimited scenario. Also, other natured-inspired techniques like variants particle swarm optimization [17] and other recent stochastic algorithms [18] can be investigated for comparative analysis.

\section{Conflict of Interests}

The authors declare that there is no conflict of interests regarding the publication of this paper.

\section{References}

[1] A. Codas and E. Camponogara, "Mixed-integer linear optimization for optimal lift-gas allocation with well-separator routing," European Journal of Operational Research, vol. 217, no. 1, pp. 222-231, 2012.

[2] T. Ray and R. Sarker, "Genetic algorithm for solving a gas lift optimization problem," Journal of Petroleum Science and Engineering, vol. 59, no. 1-2, pp. 84-96, 2007.

[3] E. Camponogara and P. H. R. Nakashima, "Solving a gas-lift optimization problem by dynamic programming," European Journal of Operational Research, vol. 174, no. 2, pp. 1220-1246, 2006.

[4] J. N. M. de Souza, J. L. de Medeiros, A. L. H. Costa, and G. C. Nunes, "Modeling, simulation and optimization of continuous 
gas lift systems for deepwater offshore petroleum production," Journal of Petroleum Science and Engineering, vol. 72, no. 3-4, pp. 277-289, 2010.

[5] M. Mahmudi and M. T. Sadeghi, "The optimization of continuous gas lift process using an integrated compositional model," Journal of Petroleum Science and Engineering, vol. 108, pp. 321327, 2013.

[6] R. Sharma, K. Fjalestad, and B. Glemmestad, "Optimization of lift gas allocation in a gas lifted oil field as non-linear optimization problem," Modeling, Identification and Control, vol. 33, no. 1, pp. 13-25, 2012.

[7] O. G. Santos, S. N. Bordalo, and F. J. S. Alhanati, "Study of the dynamics, optimization and selection of intermittent gaslift methods-a comprehensive model," Journal of Petroleum Science and Engineering, vol. 32, no. 2-4, pp. 231-248, 2001.

[8] E. P. Kanu, J. Mach, and K. E. Brown, "Economic approach to oil production and gas allocation in continuous gas lift," Journal of Petroleum Technology, vol. 33, no. 10, pp. 1887-1892, 1981.

[9] A. Ibitola and C. G. Monyei, "Genetic algorithm for students allocation to halls of residence using energy consumption as discriminant adaptive computing," Information Systems, Development Informatics \& Allied Research Journal, vol. 3, no. 4, pp. 31-36.

[10] C. G. Monyei and O. A. Fakolujo, "Optimized enhanced control system for the Unibadan's virtual power plant project using genetic algorithm," African Journal of Computing \& ICT, vol. 6 , no. 4, pp. 16-20, 2013.

[11] D. Goldberg, Genetic Algorithms in Search, Optimization and Machine Learning, Addison-Wesley, Reading, Mass, USA, 1989.

[12] A. O. Adewumi, B. A. Sawyerr, and M. M. Ali, "A heuristic solution to the university timetabling problem," Engineering Computations, vol. 26, no. 8, pp. 972-984, 2009.

[13] A. R. Yildiz and F. Ozturk, "Hybrid enhanced genetic algorithm to select optimal machining parameters in turning operation," Proceedings of the Institution of Mechanical Engineers B: Journal of Engineering Manufacture, vol. 220, no. 12, pp. 2041-2053, 2006.

[14] T. C. Nwaoha, Z. Yang, J. Wang, and S. Bonsall, "Application of genetic algorithm to risk-based maintenance operations of liquefied natural gas carrier systems," Proceedings of the Institution of Mechanical Engineers E: Journal of Process Mechanical Engineering, vol. 225, no. 1, pp. 40-52, 2011.

[15] P. Guo, X. Wang, and Y. Han, "The enhanced genetic algorithms for the optimization design," in Proceedings of the 3rd International Conference on BioMedical Engineering and Informatics (BMEI '10), pp. 2990-2994, October 2010.

[16] A. O. Adewumi and M. M. Ali, "A multi-level genetic algorithm for a multi-stage space allocation problem," Mathematical and Computer Modelling, vol. 51, no. 1-2, pp. 109-126, 2010.

[17] M. A. Arasomwan and A. O. Adewumi, "An adaptive velocity particle swarm optimization for high-dimensional function optimization," in Proceedings of the IEEE Congress on Evolutionary Computation (CEC '13), pp. 2352-2359, 2013.

[18] S. Chetty and A. O. Adewumi, "Three new stochastic local search algorithms for continuous optimization problems," Computational Optimization and Applications, vol. 56, no. 3, pp. 675721, 2013. 


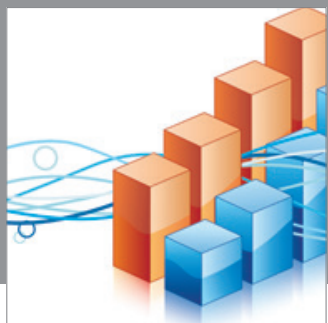

Advances in

Operations Research

mansans

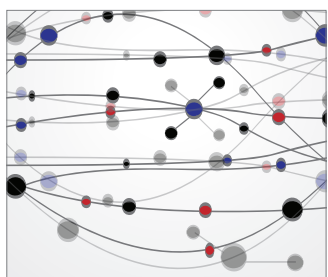

The Scientific World Journal
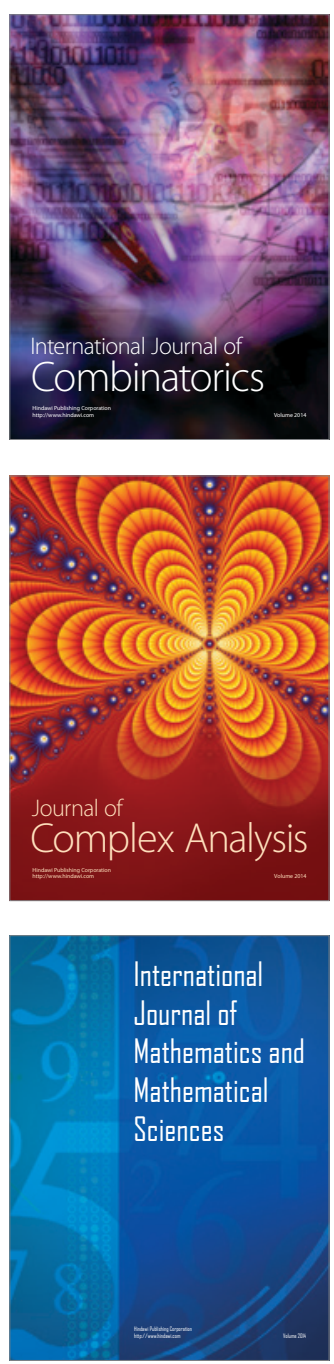
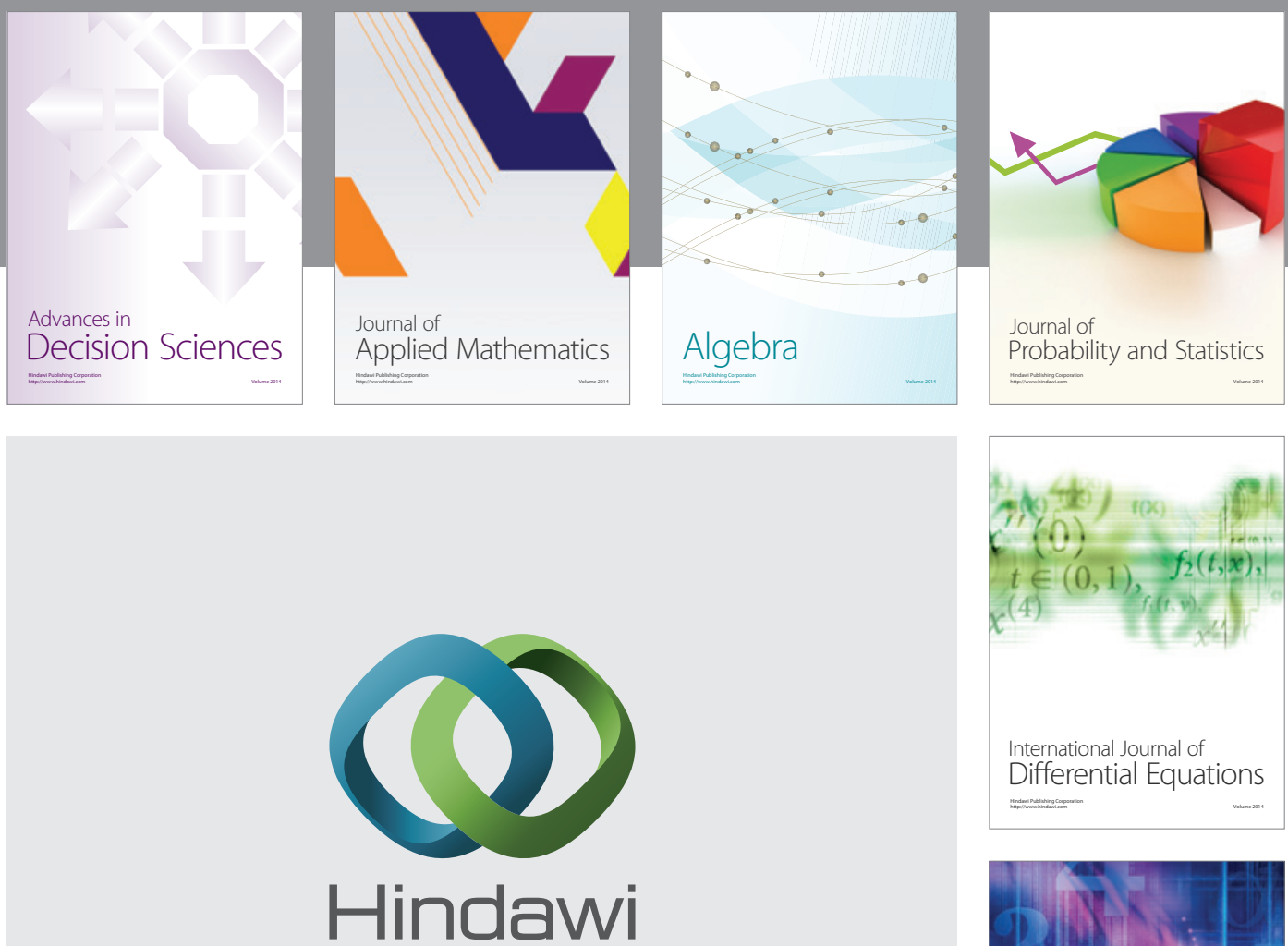

Submit your manuscripts at http://www.hindawi.com
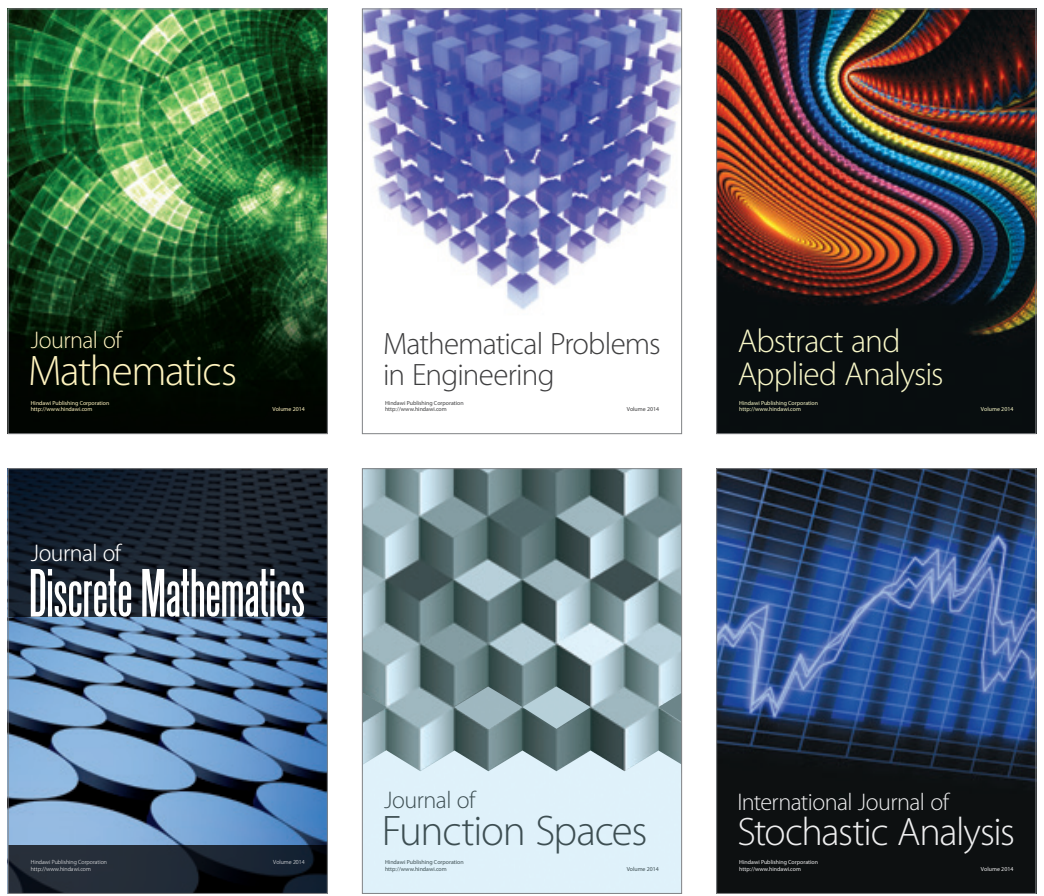

Journal of

Function Spaces

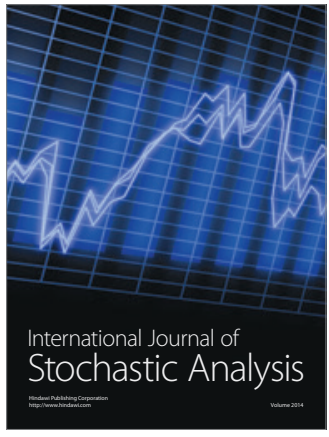

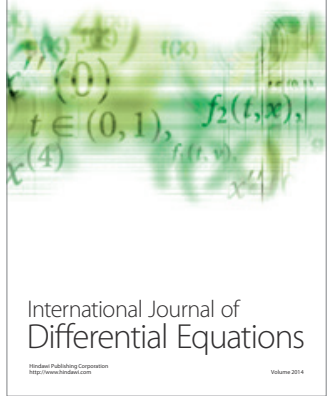
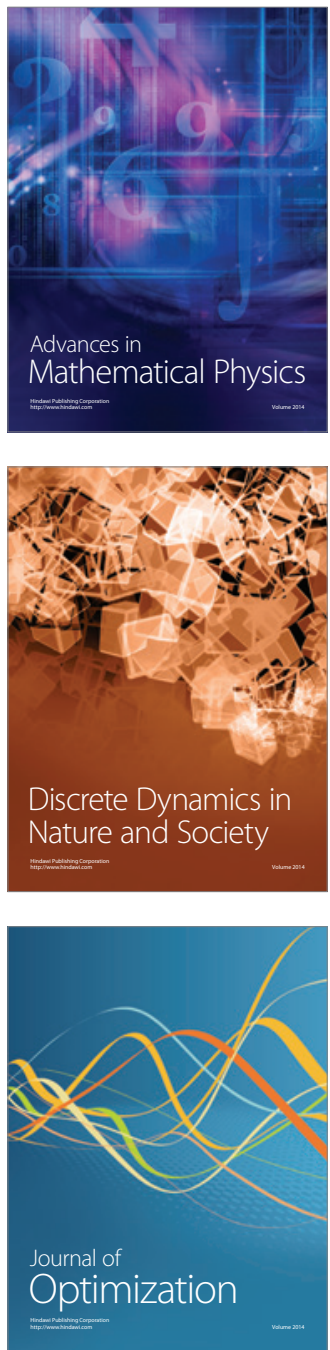